\title{
Fabricación de biocombustibles sólidos densificados (briquetas) a base de serrín de acacia y estiércol de bovinos en el departamento de Córdoba
}

\author{
Manufacture of densified solid biofuels (briquettes) made of acacia sawdust and \\ cattle manure in the region of Cordoba
}

\author{
Jorge Mario Mendoza Fandiño ${ }^{1 *} \quad$ Yahir Enrique González Doria ${ }^{1}$ \\ Miguel Doria Oviedo ${ }^{1} \quad$ Álvaro Pedroza Urueta ${ }^{1} \quad$ Alejandro Fabio Ruiz Garcés ${ }^{2}$
}

Recibido 26 de julio de 2018, aceptado 06 de febrero de 2019

Received: July 26, 2018 Accepted: February 06, 2019

\begin{abstract}
RESUMEN
En Colombia alrededor de 460.000 hogares carecen de energía eléctrica, el 4\% de la población total. El departamento de Córdoba cuenta aproximadamente con 1,9 millones de cabezas de ganado lo que anualmente se traduce a una cantidad aproximada de 4,2 millones de toneladas de estiércol y presenta un mercado emergente de aserraderos. Este trabajo evalúa las propiedades fisicoquímicas y energéticas de briquetas elaboradas con mezclas de serrín de acacia y estiércol de bovino del departamento de Córdoba. Primero se determinan porcentajes y cantidad de las mezclas de serrín-estiércol, estos se establecen mediante un diseño experimental simplex lattice. Para fabricar las briquetas se inicia con el secado y triturado de la biomasa donde la humedad final es inferior al $12 \%$ y tamaño de grano de $2 \mathrm{~mm}$, luego se compactan las mezclas en una briquetadora semiautomática a $10 \mathrm{MPa}$, luego de tener todas las briquetas fabricadas, se realizan los ensayos de resistencia a compresión y durabilidad mecánica para determinar la manejabilidad de las briquetas. Mediante el análisis próximo-elemental se calcula analíticamente el poder calorífico y las emisiones de azufre, y a través de la WBT se evalúa la eficiencia energética. Analizando los resultados de los ensayos anteriores, la mezcla más favorable es la briqueta con $60 \%$ estiércol y $40 \%$ serrín, la cual se somete a un proceso de gasificación en un gasificador Downdraft en tres posiciones de válvula, semicerrada, media y totalmente abierta, dando un poder calorífico bajo de $1556 \mathrm{~kJ} / \mathrm{kg}\left(2155 \mathrm{~kJ} / \mathrm{Nm}^{3}\right)$ y una potencia de generación de $11,8 \mathrm{~kW}$.
\end{abstract}

Palabras clave: Briqueta, estiércol, serrín.

\section{ABSTRACT}

In Colombia around 460,000 households lack of electricity, 4\% of the total population. The department of Córdoba has approximately 1.9 million livestock, which annually translates to 4.2 million tons of manure and presents an emerging market of sawmills. This work assesses the physicochemical and energetic properties of briquettes made with mixtures of acacia sawdust and cattle manure from the department of Córdoba are evaluated, carrying out tests and analytical calculations. First the sawdust-manure mixture percentage is determined, these were established by an experimental simplex lattice design. The process begins with the drying and crushing of the biomass where the final humidity is less than $12 \%$ and grain size of $2 \mathrm{~mm}$. Then the mixtures are compacted in a semi-automatic briquette machine. After, the tests of compressive strength and mechanical durability are carried out, with these the manageability

1 Departamento Ingeniería Mecánica. Universidad de Córdoba. Carrera 6 \# 76-103. Montería, Colombia. E-mail: jorge.mendoza@correo.unicordoba.edu.co

2 Departamento Ingeniería Eléctrica. Universidad del Sinú. Carrera 1W \# 38-153. Montería, Colombia. E-mail: alejandroruiz@unisinu.edu.co

* Autor de correspondencia: jorge.mendoza@ correo.unicordoba.edu.co 
of the briquettes is determined. Through the proximal-elemental analysis, the calorific value and the sulfur emissions are analytically calculated, and through the boiling water test, the energy efficiency is evaluated. Moreover, it was found the most favorable mixture in terms of the results of the previous tests, giving as outcome the briquette with $60 \%$ manure. Finally, the most favorable briquette is subjected to a gasification process in a Downdraft gasifier in three valve positions, half closed, half and fully open, in order to find the properties of the gas produced by the briquette. The result of the gas gives a CV of $1556 \mathrm{~kJ} / \mathrm{kg}$ and a generation power of $11.8 \mathrm{~kW}$.

Keywords: Briquette, manure, sawdust.

\section{INTRODUCCIÓN}

La búsqueda de la solución o mitigación a los problemas medio ambientales a nivel mundial exige que tanto los países desarrollados como los subdesarrollados creen alternativas en conjunto en el sector energético. Los combustibles fósiles siguen siendo las fuentes principales de energía global, estos están asociados con el aumento de las emisiones de dióxido de carbono $\left(\mathrm{CO}^{2}\right)$ y no se pueden renovar. Por otro lado, en Colombia alrededor de 460.000 hogares carecen de energía eléctrica, correspondiente al $4 \%$ de la población total, de estos el $70 \%$ corresponde a zonas interconectadas y el $30 \%$ a zonas no interconectadas, esto dificulta la conservación de alimentos, acceso a entretenimiento y fomenta la tala de árboles para el uso de la leña como combustible en labores diarias. El despliegue de Fuentes de Energía Renovables (RES) puede desempeñar un papel fundamental para reducir tanto las emisiones de $\mathrm{CO}^{2}$ como la dependencia de los combustibles fósiles (En la actividad ganadera el estiércol es un producto potencialmente contaminante por gases de efecto invernadero) [1]. Se estima que un animal produce en promedio $30 \mathrm{Kg} /$ día de estiércol y podría elevarse hasta $45 \mathrm{~kg} /$ día, [2] de estos un $85 \%$ es agua y la restante biomasa. Teniendo en cuenta que el departamento de córdoba cuenta con aproximadamente 1,9 millones de cabezas de ganado [3], significa una cantidad aproximada de 4,2 millones de toneladas de estiércol al año sin uso específico en la región. En los últimos años se ha fomentado en la región, la siembra de árboles maderables como el roble (quercus humboldtii), la teca (Tectona grandis), Acacia mangium y Cedro (Cedrela odorota), con interés comercial, las cuales son usadas en los aserraderos y carpinterías. El serrín, producto del aserrado de la madera de acacia no se le está dando un uso específico, este se usa en pesebreras y vehículos para transporte de animales, y representa un problema para los aserraderos por el espacio que ocupa los volúmenes que se producen diario de este material (de cada árbol que se destina a esta actividad sólo se comercializa el $20 \%$, un $40 \%$ se deja en el campo (raíces, ramas, etc.) y el $40 \%$ restante corresponde a la astilla, corteza y serrín generado durante el proceso de aserrío y de los cuales el 5\% pertenece netamente a serrín.

La energía de las biomasas es una de las fuentes de energía renovable, la biomasa se conoce como el material orgánico de origen biológico producido por las plantas, uno de los mejores métodos para aprovechar dichos residuos consiste en la aglomeración de pequeñas partículas en [5]. Para la fabricación de estas lo ideal es utilizar recursos de fácil obtención en la región donde se desea trabajar, es por esto que materiales como el estiércol de ganado bovino y el serrín de acacia se hacen atractivos como recursos para biocombustibles sólidos densificados.

La elaboración de briquetas es un método por medio del cual se puede aprovechar el poder calorífico de las biomasas, estiércol de ganado bovino y serrín de acacia propias del departamento de Córdoba como fuente de energía renovable, los cuales son 11914 $\mathrm{kJ} / \mathrm{kg}$ y $17038 \mathrm{~kJ} / \mathrm{kg}$, respectivamente.

Datos de gases obtenidos luego de llevar a cabo el proceso de combustión con briquetas de serrín en una caldera con capacidad nominal de $23,7 \mathrm{~kW}$, y la comparación con datos de la literatura científica, permitieron a [6] concluir que el uso de briquetas para la generación de calor es más beneficioso que la quema de madera (leña), debido a la menor cantidad de contaminantes, pero menos ventajosa en comparación con la quema de pellets.

Elaborando briquetas de serrín y neen en polvo, variando las presiones de compactación y los porcentajes en 
masa [7], concluye luego de varias pruebas que el rendimiento de las briquetas mejora con el alza de la presión, también en el mismo estudio concluyeron que este segundo aportaba durabilidad, buenas características al momento de transporte y manipulación, y el serrín mejoraba el poder calorífico de las briquetas.

Este proyecto tiene como propósito la fabricación de biocombustibles solidos densificados utilizando materiales presentes en el departamento de Córdoba, serrín de acacia y estiércol de ganado bovino, que aporten su poder calorífico en procesos de combustión como hornos, estufas, calderas, y favorables características de manipulación y transporte, como una alternativa para mitigar el impacto ambiental por gases, fomentar la creación y uso de energías renovables y buscar un método adecuado para proveer de energía eléctrica a las zonas rurales de Colombia que no cuentan con este servicio.

\section{MATERIALES Y MÉTODOS}

La elaboración de las briquetas se inició con la preparación de las biomasas, mediante los procesos de secado, triturado y tamizado. Lo primero que se realizó fue el secado de la biomasa de forma natural, luego se redujo el tamaño de grano de la biomasa a $2 \mathrm{~mm}$ por medio de una trituradora de molino de martillos, modelo TP-24, número de serie 3458 , de 3500 a $3600 \mathrm{rpm}$ del motor y cuenta con una potencia nominal de $10 \mathrm{HP}$. Al terminar el proceso de molienda se inició el tamizado, el cual es necesario para que haya uniformidad entre partículas y así lograr una mayor superficie de contacto en el proceso de briquetado, en consecuencia, mayor densidad y durabilidad de las briquetas [8]; es por esto que el tamaño de partícula tanto del estiércol como del serrín fueron estandarizados mediante tamices de $2 \mathrm{~mm}$ de abertura bajo la norma ASME E-11/2004, para así garantizar el tamaño de grano deseado.

Luego de tener la biomasa preparada y teniendo en cuenta que las briquetas se van a realizar con dos biomasas, estiércol de ganado bovino y serrín de acacia, se varían los porcentajes como se muestra en la Tabla 1, puesto que lo que se busca es ver el efecto de cada componente de la mezcla en las emisiones de azufre, poder calorífico, eficiencia energética, resistencia a la compresión y durabilidad mecánica. No se realiza una optimización debido a la simplicidad del diseño de experimento, donde la única variable es la concentración en masa, la cual una depende de la otra.

Una vez preparadas las mezclas se procede a la fabricación de las briquetas, la cual se llevó a cabo en una máquina briquetadora semiautomática GEMCO ubicada en el laboratorio de energías renovables de la UPB sede Montería, esta máquina tiene una potencia de motor de $30 \mathrm{hp}$, con un diámetro de matriz de $50 \mathrm{~mm}$, tipo YX3-200L 1-6, ejerce altas presiones y temperaturas entre 50 a $250{ }^{\circ} \mathrm{C}$ para realizar el proceso de compactación (ver Figura 1).

Como se puede ver en la Tabla 1 , con $3 \mathrm{~kg}$ de mezcla se elaboraron 10 briquetas variando los porcentajes,

Tabla 1. Porcentaje de mezclas.

\begin{tabular}{|c|c|c|c|c|}
\hline Estiércol de ganado bovino $(\boldsymbol{\%})$ & Serrín de acacia $(\boldsymbol{\%})$ & Cantidad (unidades) & Estiércol (kg) & Serrín $(\mathbf{k g})$ \\
\hline 0 & 100 & 10 & 3 & 0 \\
\hline 10 & 90 & 10 & 2,7 & 0,3 \\
\hline 20 & 80 & 10 & 2,4 & 0,6 \\
\hline 30 & 70 & 10 & 2,1 & 0,9 \\
\hline 40 & 60 & 10 & 1,8 & 1,2 \\
\hline 50 & 50 & 10 & 1,5 & 1,5 \\
\hline 60 & 40 & 10 & 1,2 & 1,8 \\
\hline 70 & 30 & 10 & 0,9 & 2,1 \\
\hline 80 & 20 & 10 & 0,6 & 2,4 \\
\hline 90 & 10 & 10 & 0,3 & 2,7 \\
\hline 100 & 0 & 10 & 0 & 3 \\
\hline
\end{tabular}

Fuente: Elaboración Propia. 
Fuente: Elaboración Propia.

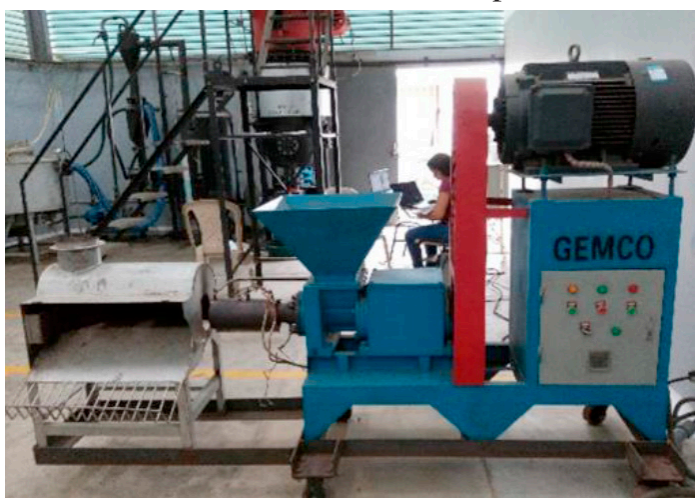

Figura 1. Máquina briquetadora.

para así obtener el número de briquetas necesarias teniendo en cuenta la posterior realización de los ensayos de compresión, durabilidad mecánica y WBT.

Después de elaboradas las briquetas, estas fueron sometidas a pruebas de resistencia a la compresión, durabilidad mecánica y WBT, con el fin de determinar la manejabilidad de estas y la eficiencia energética. El ensayo de resistencia a la compresión se realizó por medio de la máquina universal de ensayos MTS Criterion modelo 45, con capacidad de carga de 300 $\mathrm{KN}$ a una presión de $20 \mathrm{MPa}$ y bajo la norma técnica colombiana NTC 2060, briquetas combustibles para uso doméstico. Se trabajó con una carga de $3 \mathrm{kN} /$ min a varias muestras elegidas aleatoriamente y de acuerdo a la masa de cada espécimen debe tener cierta resistencia a la compresión (ver Figura 2).

Luego se llevó a cabo la prueba de durabilidad mecánica, esta se realizó en un equipo para ensayos de durabilidad mecánica, la cual consiste en someter las briquetas a golpes entre sí y con las paredes de una caja rotativa, bajo los parámetros establecidos en la norma CEN/TS 15210. Para este ensayo se introdujeron dos briquetas dentro de la caja rotativa, luego se hace girar a $21 \mathrm{rpm}$ por 120 segundos.

Se realizaron también cálculos analíticos de poder calorífico y emisiones de azufre, mediante los datos obtenidos del análisis próximo, concernientes al poder calorífico y contenido de azufre de las biomasas, estiércol de ganado bovino y serrín de acacia.

El poder calorífico se calculó mediante la suma del producto del poder calorífico de las biomasas,
Fuente: Elaboración Propia.

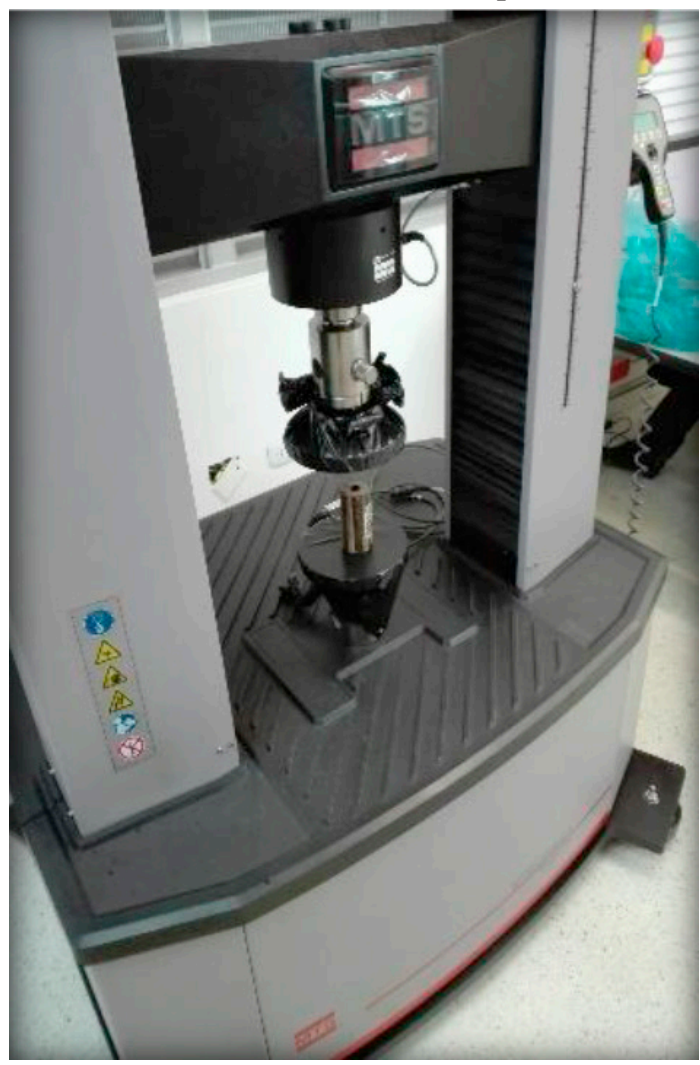

Figura 2. Montaje ensayo a compresión.

el porcentaje en la mezcla y la densidad de cada biomasa, dividido entre la suma del producto de la densidad de cada biomasa y el porcentaje en la mezcla, resumido en la ecuación 1 .

$$
P C=\frac{(p c s . \rho s . \% s)+(\text { pce. } . \rho e . \% e)}{(\rho s . \% s)+(\rho e . \% e)}
$$

Donde:

PC: Poder calorífico de la mezcla

pcs: Poder calorífico del serrín

$\%$ s: Porcentaje en masa del serrín

$\rho$ : Densidad del serrín

pce: Poder calorífico del estiércol.

\%: Porcentaje en masa del estiércol

pe: Densidad del estiércol

El contenido de azufre se calculó mediante la suma del producto del contenido de azufre de las biomasas, el porcentaje en la mezcla y la densidad de cada 
biomasa, dividido entre la suma del producto de la densidad de cada biomasa y el porcentaje en la mezcla, resumido en la ecuación 2 .

$$
\% S=\frac{(S s . \rho s . \% s)+(\text { Se. } \rho e . \% e)}{(\rho s . \% s)+(\rho e . \% e)}
$$

Donde:

\%: $\quad$ Porcentaje de azufre de la mezcla

Ss: $\quad$ Contenido de azufre del serrín

\%s: $\quad$ Porcentaje en masa del serrín

os: $\quad$ Densidad del serrín

$\mathrm{Se}$ : Contenido de azufre del estiércol

\%e: $\quad$ Porcentaje en masa del estiércol

คe: $\quad$ Densidad del estiércol

La WBT o prueba de agua hirviendo fue hecha para los parámetros determinado por bailish y fue de tipo alta potencia en frio.

Los siguientes parámetros pudieron ser obtenidos durante la prueba:

\section{Tiempo de encendido:}

Es el tiempo que demora la briqueta en encender completamente.

\section{Tiempo de combustion:}

Es el tiempo que demora la briqueta en llevar el agua de la temperatura ambiente a su temperatura de ebullicion.

\section{Velocidad de combustión (g/min):}

Es la medida del rango de consumo de combustible para lograr hervir el agua en un recipiente. Es calculado dividiendo el equivalente del combustible consumido entre el tiempo de prueba.

\section{Eficiencia térmica:}

$$
P U H=\frac{M w \cdot C p \cdot(T b-T o)+M c \cdot L}{M f \cdot E f} \times 100 \%
$$

Donde:

PHU $=$ Porcentaje de calor utilizado;

$\mathrm{P}=\quad$ Potencia de salida;

S.F.C = Consumo específico de combustible;

B.R = Tasa de combustión;

$\mathrm{Mw}=$ Masa de agua en la maceta $(\mathrm{kg}) ;$
$\mathrm{Cp}=$ Calor específico del agua $(\mathrm{kJ} / \mathrm{kgK})$;

$\mathrm{A}=\quad$ temperatura inicial del agua $(\mathrm{K})$;

$\mathrm{Tb}=$ Temperatura de ebullición del agua $(\mathrm{K})$;

$\mathrm{Mc}=$ masa de agua evaporada $(\mathrm{kg}) ;$

$\mathrm{L}=\quad$ calor de evaporación latente $(\mathrm{kg})$;

$\mathrm{Mf}=$ masa de combustible quemado $(\mathrm{kg})$;

$\mathrm{Ef}=\quad$ Valor calorífico del combustible $(\mathrm{kJ} / \mathrm{kg})$;

$\mathrm{t}=\quad$ tiempo necesario para quemar combustible (segundos).

Luego de tener todos los resultados de los ensayos y cálculos analíticos anteriormente mencionados, se determinó la mezcla más favorable. Para esto se realizó un diseño de experimento bajo un escenario de optimización mediante el software Design Expert versión 6, con el modelo Simplex-lattice. Se hizo una optimización de múltiples objetivos con el fin de determinar la mejor formulación en términos de las variables de decisión, es decir, la mezcla más favorable para elaborar briquetas teniendo en cuenta los resultados de los ensayos y cálculos analíticos a los cuales fueron sometidas estas, tales como resistencia a la compresión, durabilidad mecánica, WBT, poder calorífico y porcentaje de azufre. Aquí se les dio valor a las variables tenidas en cuenta, de acuerdo a la experiencia y la importancia que estas tienen en las briquetas, se maximizaron algunas variables como eficiencia energética de la WBT y poder calorífico, mientras otras se mantuvieron en todo su rango de aceptabilidad como durabilidad mecánica, resistencia a la compresión y azufre. Con las superficies de respuesta se puede apreciar la interacción de los factores en las variables de respuesta.

Luego de obtener la mezcla más favorable, se utilizó la misma para el proceso de gasificación, esta fue realizada en un gasificador ANKUR tipo Downdraft modelo WBG-60 (GAS32) con capacidad de $200 \mathrm{~kg}$. Se inició con el encendido a carbón de la llama y una medida de cromatografía para el gas patrón, luego de esto se procede al vertimiento de $52 \mathrm{~kg}$ de la briqueta seleccionada al cilindro del gasificador. Para realizar las mediciones en la que se hizo la prueba se tomaron 3 posiciones de válvula; semicerrada, media y totalmente abierta, en cada una se tomaron dos mediciones de temperatura del gas, al inicio y al final del sistema de gasificación, así como la velocidad de admisión de aire. Luego de terminado el proceso se pesa la ceniza restante en el cilindro. 
Finalizado el proceso de gasificación, se procedió a extraer y analizar los datos arrojados por el cromatógrafo. Se determinaron las concentraciones de los componentes del gas, tales como $\mathrm{H}_{2}, \mathrm{O}_{2}, \mathrm{~N}_{2}$, $\mathrm{CO}, \mathrm{CH}_{4}$ y $\mathrm{CO}_{2}$, luego con estos datos se calcula el poder calorífico del gas de las briquetas.

Finalmente se obtuvo la potencia de generación del gas, primero se usó el principio de Carnot, calculando la eficiencia térmica, se asumió que el proceso se llevó a cabo bajo las siguientes condiciones, $25^{\circ} \mathrm{C}$, 1 atm de presión, y que la temperatura y presión fueron constantes durante todo el proceso, el cálculo se realizó con ayuda del software Engineering Equation Solver (EES), debido a su capacidad para la resolución e iteración de sistemas de ecuaciones complejas.

\section{RESULTADOS}

De acuerdo con la Tabla 2 y teniendo en cuenta lo mencionado por [9] se pudo determinar por el contenido de humedad que son similares en las dos biomasas, esta implica que, a mayor contenido de humedad, mayor es la cantidad de energía que debe ser destinada para este propósito. Con respecto al material volátil se observa que el estiércol tiene un 63,3\% al valor del serrín, el cual es de 70,9\%, que es un resultado muy parecido al encontrado por [10], esto mostró que el primero tiene mejores propiedades de encendido que el segundo, lo que quiere decir que el serrín libera la energía más fácilmente comparado con el estiércol [11]. Los resultados del porcentaje de ceniza muestran al estiércol con un porcentaje mucho mayor al serrín, es decir, el estiércol es menos eficiente en términos energéticos, debido a que el porcentaje de cenizas indica la cantidad de material solido no combustible por kilogramo de biomasa [12]. El Poder Calorífico Inferior (PCI) muestra que el serrín tiene $17038 \mathrm{~kJ} /$ $\mathrm{kg}$ y es un $43 \%$ mayor al del estiércol $11914 \mathrm{~kJ} / \mathrm{kg}$, por lo que las briquetas con mayor contenido de serrín se espera que tengan mayor poder calorífico. El contenido de azufre de las biomasas es inferior al máximo que puede tener una briqueta y que establece la norma NTC 2060 Briquetas Combustibles Para Uso Doméstico en $1 \%$, valor que es muy superior al obtenido del serrín y el estiércol con valores de $0,06 \%$ y $0,26 \%$ respectivamente.

\section{Fabricación de las briquetas}

Las altas presiones y temperaturas de operación de la maquina briquetadora con un diámetro de matriz de $50 \mathrm{~mm}$, hacen liberar la lignina presente en el serrín de la madera de acacia, lo que actúa como aglomerante, y da por finalizada la compactación de estas. La máquina expulsa 1,37 metros de briquetas en un minuto, las cuales son cortadas a $10 \mathrm{~cm}$ de longitud, para un total aproximado de 13 briquetas completas. Para cada mezcla de $3 \mathrm{~kg}$ se calculan perdidas máximas de masa durante el proceso del $16 \%$, lo que se ajusta a la cantidad de briquetas necesarias para los ensayos posteriores, durabilidad mecánica, resistencia a la compresión y la WBT.

En la maquina briquetadora usada, no pudieron ser elaboradas las mezclas con porcentajes de serrín superiores al $50 \%$, debido a que el punto de ignición del serrín es bajo, ocasionaba su quema dentro de la matriz, posterior atascamiento y el proceso no se llevaba a cabo de forma normal, por lo tanto, se fabricaron 6 mezclas, $50 \mathrm{E}, 60 \mathrm{E}, 70 \mathrm{E}, 80 \mathrm{E}, 90 \mathrm{E}$ y $100 \mathrm{E}$, una cantidad de 66 briquetas.

\section{Durabilidad mecánica}

Los resultados de las pruebas de durabilidad mecánica o resistencia a la abrasión, realizadas a las briquetas elaboradas con estiércol de ganado bovino y serrín de acacia. Todas las muestras se encuentran en el rango más alto de durabilidad $\mathrm{DU} \geq 95 \%$, donde cada prueba realizada arrojó resultados mayores a $99 \%$, podemos notar que no hay variabilidad

Tabla 2. Análisis próximo y elemental.

\begin{tabular}{|l|c|c|c|c|c|c|}
\hline \multicolumn{1}{|c|}{ Muestra } & HR (\%) & Ceniza (\%) & MV (\%) & Cf $(\%)$ & S (\%) & PCI kJ/kg \\
\hline Serrín & 12,09 & 1,27 & 70,92 & 15,72 & 0,06 & 17038 \\
\hline Estiércol & 11,78 & 31,71 & 44,76 & 11,75 & 0,26 & 11914 \\
\hline
\end{tabular}

Fuente: Elaboración Propia.

HR: humedad residual; MV: material volátil; Cf: carbono fijo; S: azufre PC: poder calorífico inferior; C: carbono; H: hidrogeno; N: nitrógeno. 
considerable en cada una de las pruebas realizadas para las distintas mezclas.

Estos resultados se deben en gran parte a la capacidad de compactado de la maquina briquetadora, esta ejerce altas presiones y temperaturas hasta de $250{ }^{\circ} \mathrm{C}$ lo cual hace que se libere la lignina de las biomasas y permite la unión entre partículas. Los factores anteriormente mencionados permiten concluir que todas las briquetas soporten las condiciones normales de almacenamiento y transporte.

\section{Resistencia a la compresión}

Teniendo en cuenta la Figura 3, se puede notar que todas las muestras ensayadas están considerablemente por encima de lo establecido en la norma NTC 2060. El ensayo número uno, correspondiente a la briqueta con mezcla $70 \%$ estiércol - 30\% serrín, fue la que más soporto carga antes de la fractura, $32 \mathrm{kN}$ y la que menos soporto corresponde al ensayo número nueve, $60 \%$ estiércol - $40 \%$ serrín, $9,5 \mathrm{kN}$. Los rangos relativamente grandes en las desviaciones estándar de algunas muestras, se debió a la dificultad del montaje de las briquetas en la máquina de ensayos, debido a los fenómenos de torsión y flexión que sufren estas al momento de su elaboración (ver Figura 4). Sin embargo, se puede concluir que, al aumentar el porcentaje de serrín en las briquetas, la carga máxima de fractura disminuye, mientras que el estiércol aumenta esta.

\section{Poder calorífico}

Los resultados fueron muy consecuentes con lo esperado como se puede ver en la Figura 5, en la cual se puede apreciar la linealidad de los resultados del poder calorífico, es decir, entre más contenido

Fuente: Elaboración Propia.

Resumen de gráficas ensayo de compresión

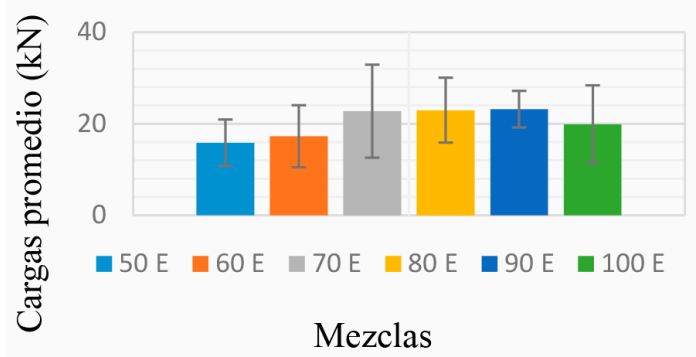

Figura 3. Resultado ensayo de compresión.
Fuente: Elaboración Propia.

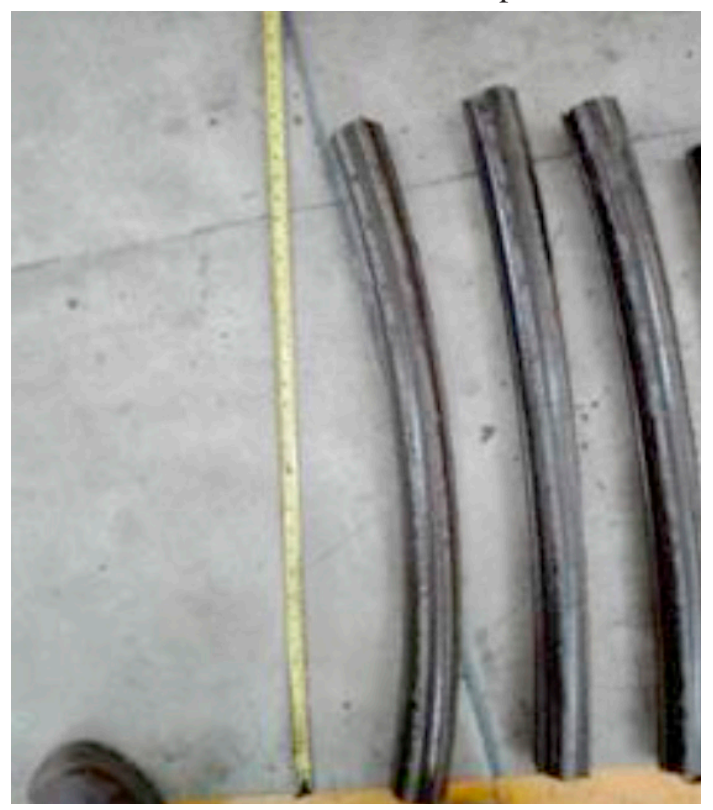

Figura 4. Flexión y torsión en briquetas.

Fuente: Elaboración Propia.

Poder calorífico de las briquetas

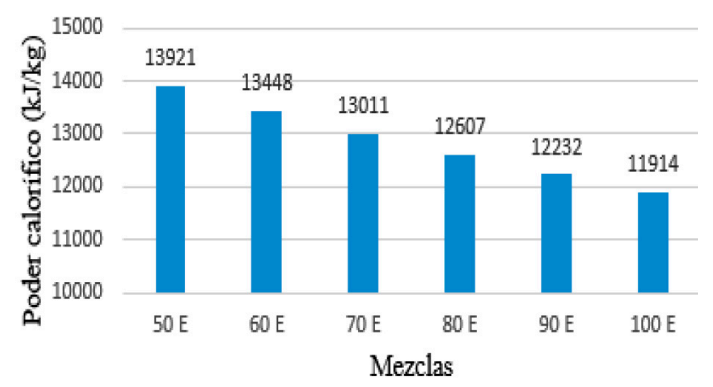

Figura 5. Resultados poder calorífico.

de serrín presente en la mezcla, mayor será el poder calorífico de la briqueta. Cabe resaltar que las briquetas con porcentajes de $90 \%$ y $100 \%$ de estiércol están por debajo del mínimo requerido por la norma NTC 2060 para briquetas no hechas a base de carbón que es de $12500 \mathrm{~kJ} / \mathrm{kg}$, sin embargo, los otros ensayos fueron realizados para analizar su comportamiento.

\section{Contenido de azufre}

En la Tabla 3 se puede observar que todas las mezclas se encuentran por debajo del $1 \%$, lo que quiere decir que todas son aceptadas teniendo en 
Tabla 3. Contenido de azufre por mezcla.

\begin{tabular}{|c|c|c|}
\hline \multicolumn{3}{|c|}{ Porcentaje de Azufre de las Briquetas } \\
\hline Estiércol (\%) & Serrín (\%) & S (\%) \\
\hline 50 & 50 & 0,16 \\
\hline 60 & 40 & 0,18 \\
\hline 70 & 30 & 0,2 \\
\hline 80 & 20 & 0,22 \\
\hline 90 & 10 & 0,235 \\
\hline 100 & 0 & 0,25 \\
\hline
\end{tabular}

Fuente: Elaboración Propia.

cuenta la norma NTC 2060 para briquetas de uso doméstico, el cual está presente en el $\mathrm{SO}_{2}$ como uno de los compuestos perjudiciales para la salud humana y el ambiente [13].

\section{Prueba de agua hirviendo (WBT)}

\section{Tiempo de encendido total del combustible}

Como se puede apreciar en la Figura 6 a medida que aumenta el contenido de estiércol, aumenta el tiempo que demora en encender completamente briqueta. El resultado es consecuente ya que el estiércol tiene menor carbono fijo y material volátil que el serrín, además del trabajo realizado por [10] donde elaboró briquetas con mezclas de diferentes proporciones de materiales como estiércol, pastos, serrín, acícula de pino y sometiéndolas a la WBT, concluyendo así que las briquetas con mayor contenido de estiércol tienen menos espacio entre partículas, por lo que hay menos oxígeno y dificulta la combustión.

\section{Tiempo de combustión}

Se aprecia en la Figura 7 la relación, entre mayor contenido de estiércol, mayor tiempo le toma a la briqueta llevar al agua a su temperatura de ebullición, debido a que el estiércol no libera la energía tan rápido

Fuente: Elaboración Propia.

Tiempo de encendido de las briquetas

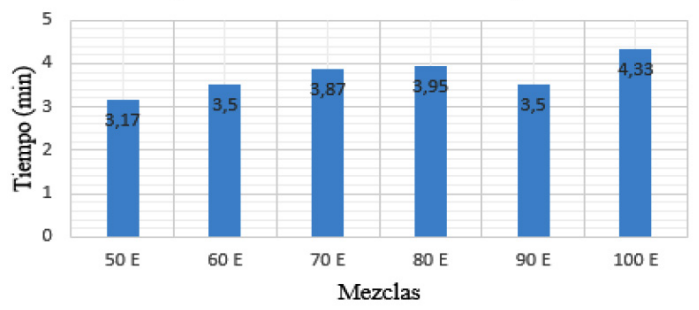

Figura 6. Resultados tiempo de encendido WBT.
Fuente: Elaboración Propia.

Tiempo de combustión de briquetas

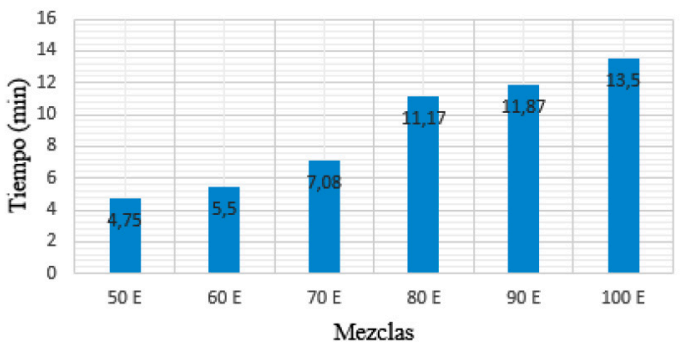

Figura 7. Resultados tiempo de combustión WBT.

como el serrín, lo que corrobora el estudio hecho por [14], que las briquetas de $100 \%$ o porcentajes altos de estiércol tienen baja circulación de aire por lo que tienen mala combustión, también se debe al mayor poder calorífico del serrín comparado con el de estiércol. Se puede notar que los resultados muestran un comportamiento lineal.

\section{Masa de agua evaporada}

Cuando se analiza la Figura 8 se observa la masa remanente de para cada briqueta, teniendo en cuenta que para todos los casos la masa inicial fue de $1 \mathrm{~kg}$. Se puede deducir que no hay mucha variación de los resultados entre las mezclas de $50 \mathrm{E}$ a $70 \mathrm{E}$, pero cuando se observan las mezclas de $80 \mathrm{E}$ hasta $100 \mathrm{E}$ se presenta una variación considerable, esto es resultado de un mayor tiempo de combustión, ya que a estas últimas mezclas les toma más tiempo superar la temperatura de ebullición del agua que las tres (3) primeras mezclas debido a su menor poder calorífico y por ende menor capacidad de superar el calor latente del agua (en general), pero se evapora en ciertos puntos de la olla en especial

Fuente: Elaboración Propia.

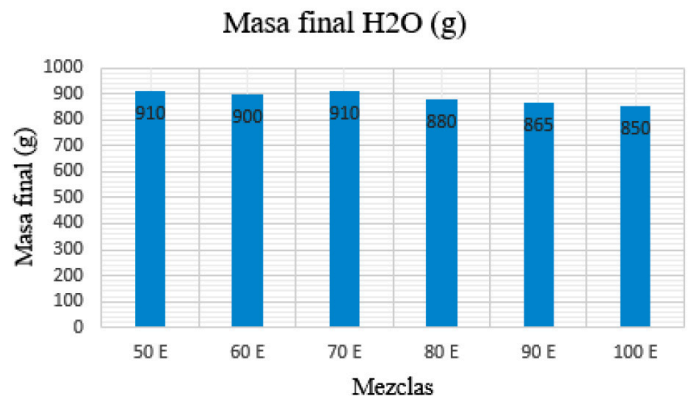

Figura 8. Masa de agua restante WBT. 
en las orillas donde alcana una temperatura mayor al resto de la olla, es por esto que al durar más tiempo se pierde más masa.

\section{Masa de combustible quemada}

Los resultados que se pueden apreciar en la Figura 9 muestran una pendiente negativa, es decir, entre mayor contenido de estiércol presente en la mezcla hubo mayor pérdida de masa en la briqueta. Teniendo en cuenta que el tiempo de combustión fue mayor en las briquetas de mayor concentración de estiércol.

\section{Velocidad de combustión}

Este resultado respalda el análisis realizado de la masa de combustible quemada, puesto que en la Figura 10 se aprecia una pendiente descendente de la mezcla $50 \mathrm{E}$ a la de $100 \mathrm{E}$, entre mayor contenido de estiércol menor es la velocidad de combustión, concluyendo así que las partículas de estiércol se consumen con menor rapidez a las de serrín, debido

Fuente: Elaboración Propia. Masa final briquetas $(\mathrm{g})$

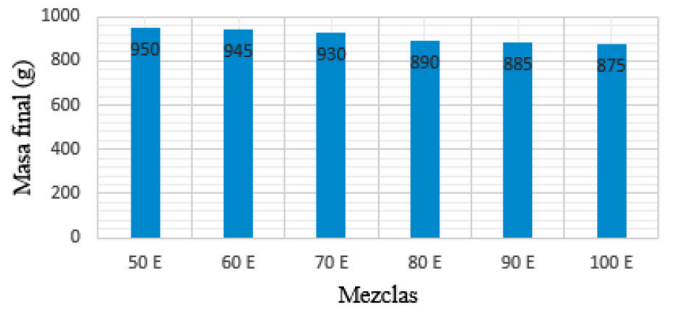

Figura 9. Resultados masa combustible consumida WBT.

Fuente: Elaboración Propia.

Velocidad de combustión de las briquetas

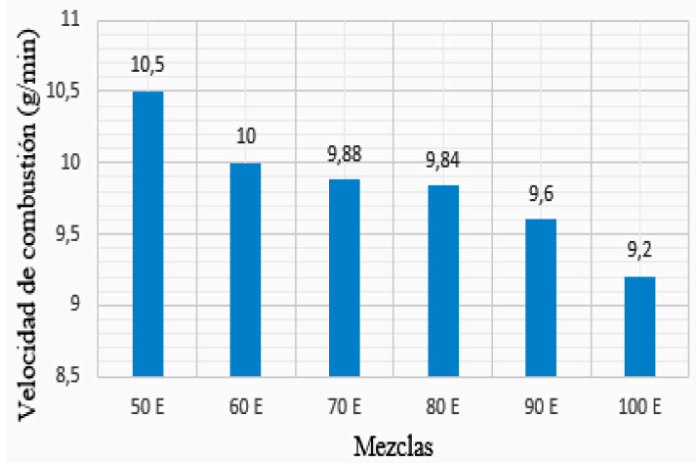

Figura 10. Resultados velocidad de combustión WBT. a que este último tiene mayor carbono fijo y material volátil que facilita la combustión.

\section{Eficiencia energética}

Como se puede apreciar en la Figura 11 y usando la ecuación 3 las briquetas con mayor eficiencia son las de $50 \mathrm{E}$ y $60 \mathrm{E}$, a partir de ahí la pendiente decrece hasta $100 \mathrm{E}$. Se deduce de esta figura que las partículas de serrín liberan con mayor facilidad su energía que las de estiércol, además de su mayor poder calorífico. Por lo que se puede sacar mejor provecho de las briquetas con mayor porcentaje de serrín, esto se ve reflejado en el dato el cual una briqueta de $50 \mathrm{E}$ da lo que brinda 3 de $100 \mathrm{E}$.

Por otro lado, hay otro aspecto que afecta la eficiencia energética y es la densidad de las briquetas, lo cual afecta especialmente a las briquetas con alto contenido de estiércol, que al estar muy comprimidas impiden la circulación de oxígeno, por lo que disminuye la capacidad de una buena combustión.

\section{Determinación de la mezcla favorable}

En la primera optimización se maximizó el contenido de estiércol, el poder calorífico y la eficiencia energética, se mantuvo la resistencia a la compresión en su rango mínimo y máximo y el contenido de serrín en un rango de 0 y $50 \%$, también se le dio una importancia a cada variable, (ver Tabla 4). El software recomienda teniendo en cuenta los datos de entrada, sus rangos y la importancia para cada uno de ellos, que la mezcla con las mejores características es la de 56\% de estiércol y $44 \%$ de serrín y las características de esta se muestran en la Tabla 5. De las briquetas elaboradas la que más

Fuente: Elaboración Propia.

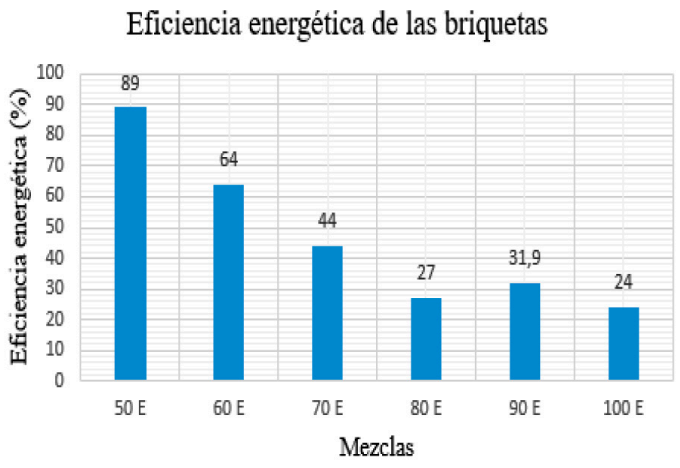

Figura 11. Resultados eficiencia energética WBT. 
Tabla 4. Resumen de resultados por mezcla.

\begin{tabular}{|c|c|c|c|c|c|c|c|}
\hline \multicolumn{7}{|c|}{ Tabla de resultados de ensayos a briquetas de Serrín y Estiércol } \\
\hline $\begin{array}{c}\text { Estiércol } \\
(\boldsymbol{\%})\end{array}$ & $\begin{array}{c}\text { Serrín } \\
(\boldsymbol{\%})\end{array}$ & $\begin{array}{c}\text { Resistencia a la } \\
\text { compresión }(\mathbf{N})\end{array}$ & $\begin{array}{c}\text { Poder calorífico } \\
(\mathbf{k J} / \mathbf{k g})\end{array}$ & $\begin{array}{c}\text { Durabilidad } \\
\text { mecánica }(\boldsymbol{\%})\end{array}$ & $\begin{array}{c}\text { Eficiencia } \\
\text { combustión } \mathbf{( \% )})\end{array}$ & $\begin{array}{c}\text { Azufre } \\
(\boldsymbol{\%})\end{array}$ & $\begin{array}{c}\text { Cenizas } \\
(\boldsymbol{\%})\end{array}$ \\
\hline 0,5 & 0,5 & 15878 & 13921 & 99,58 & 89 & 0,16 & 16,27 \\
\hline 0,6 & 0,4 & 17267 & 13448 & 99,80 & 64 & 0,18 & 19,7 \\
\hline 0,7 & 0,3 & 22747 & 13011 & 99,44 & 44 & 0,2 & 22,1 \\
\hline 0,8 & 0,2 & 22974 & 12607 & 99,81 & 27 & 0,22 & 26,8 \\
\hline 0,9 & 0,1 & 23186 & 12232 & 99,29 & 31,9 & 0,235 & 29,3 \\
\hline 1 & 0 & 19926 & 11914 & 99,30 & 24 & 0,25 & 31,27 \\
\hline
\end{tabular}

Fuente: Elaboración Propia.

Tabla 5. Parámetros optimización 4.

\begin{tabular}{|l|l|c|c|c|c|c|}
\hline \multirow{2}{*}{ Nombre } & \multirow{2}{*}{ Meta } & Mínimo & Máximo & Mínimo & Máximo & \multirow{2}{*}{ Importancia } \\
\cline { 3 - 6 } & & Limite & Limite & \multirow{2}{*}{ Peso } & \multirow{2}{*}{ Peso } & \\
\hline Estiércol & Maximizar & 0,5 & 1 & 1 & 1 & 3 \\
\hline Serrín & En rango & 0 & 0,5 & 1 & 1 & 3 \\
\hline Poder Calorífico & Maximizar & 11914 & 13921 & 1 & 1 & 3 \\
\hline R Compresión & En rango & 15878 & 23186 & 1 & 1 & 3 \\
\hline Eficiencia energética & Maximizar & 24 & 89 & 1,71355 & 1 & 5 \\
\hline
\end{tabular}

Fuente: Elaboración Propia.

se aproxima a este resultado es la de $60 \%$ estiércol - $40 \%$ serrín, por lo cual se convierte en la mezcla favorable de las elaboradas.

En la Tabla 6, se hizo una optimización de múltiples objetivos, pero este con el fin de determinar las mejores formulaciones en términos de las variables de decisión, es decir, el rango de mezclas más favorables para elaborar briquetas teniendo en cuenta los resultados de los ensayos y cálculos analíticos a los cuales fueron sometidas estas, se buscaban rangos de mezclas que tuvieran la máxima cantidad de estiércol, sin sacrificar variables como la eficiencia energética y el PCI, se tuvo en cuenta para realizar esta optimización que en la región este tipo de biomasa es abundante. De igual forma se le dieron valores de importancia a las variables, se aumentó el contenido de estiércol entre $60=90 \%$, serrín entre $0-50 \%$, el rango de poder calorífico entre 12500 y 13921 y eficiencia energética entre 50 y 89 como se puede ver en la Tabla 7. El software recomienda cuatro combinaciones, las cuales se pueden ver en la Tabla 8.

\section{Gasificación}

En la Tabla 9 se pueden observar las concentraciones del gas de la briqueta para las distintas posiciones de válvula, se aprecia que el mayor contenido de Nitrógeno se presenta con la válvula totalmente abierta, resultado que está acorde a lo dicho por [15]

Tabla 6. Resultados optimización I.

\begin{tabular}{|c|c|c|c|c|c|}
\hline \multicolumn{5}{|c|}{ Solución } \\
\hline Nombre & Estiércol (\%) & Serrín (\%) & $\begin{array}{c}\text { Poder calorífico } \\
\text { (kJ/kg) }\end{array}$ & $\begin{array}{c}\text { Resistencia a la } \\
\text { compresión (N) }\end{array}$ & $\begin{array}{c}\text { Eficiencia } \\
\text { energética (\%) }\end{array}$ \\
\hline & 56 & 44 & 13644 & 17606,8 & 73,34 \\
\hline
\end{tabular}

Fuente: Elaboración Propia. 
Tabla 7. Parámetros de optimización II.

\begin{tabular}{|l|c|c|c|c|c|c|}
\hline \multirow{2}{*}{ Nombre } & \multirow{2}{*}{ Meta } & Mínimo & Máximo & Mínimo & Máximo & \multirow{2}{*}{ Importancia } \\
\cline { 3 - 6 } & & Limite & Limite & Peso & Peso & \\
\hline Estiércol & En rango & 0,6 & 0,9 & 1 & 1 & 3 \\
\hline Serrín & En rango & 0 & 0,5 & 1 & 1 & 3 \\
\hline Poder Calorífico & En rango & 12500 & 13921 & 1 & 1 & 3 \\
\hline R Compresión & En rango & 15878 & 23186 & 1 & 1 & 3 \\
\hline Eficiencia energética & En rango & 50 & 89 & 1,71355 & 1 & 5 \\
\hline
\end{tabular}

Fuente: Elaboración Propia.

Tabla 8. Resultados optimización II.

\begin{tabular}{|c|c|c|c|c|c|}
\hline \multicolumn{7}{|c|}{ Solución } \\
\hline Nombre & $\begin{array}{c}\text { Estiércol } \\
(\boldsymbol{\%})\end{array}$ & $\begin{array}{c}\text { Serrín } \\
(\boldsymbol{\%})\end{array}$ & $\begin{array}{c}\text { Poder calorífico } \\
(\mathbf{k J} / \mathbf{k g})\end{array}$ & $\begin{array}{c}\text { Resistencia a la } \\
\text { compresión (N) }\end{array}$ & $\begin{array}{c}\text { Eficiencia } \\
\text { energética (\%) }\end{array}$ \\
\hline A & 63 & 37 & 13302 & 20245 & 58,4 \\
\hline B & 62 & 38 & 13339 & 19996 & 57,1 \\
\hline C & 61 & 39 & 13416 & 19452 & 61,7 \\
\hline D & 66 & 34 & 13191 & 20945 & 51,45 \\
\hline
\end{tabular}

Fuente. Elaboración Propia.

Tabla 9. Resultados del Syngas.

\begin{tabular}{|c|c|c|c|c|}
\hline \multicolumn{5}{|c|}{ Cromatografía Briquetas } \\
\hline Componente & $\begin{array}{c}\text { Conc. Gas } \\
\text { Patrón (\%) }\end{array}$ & $\begin{array}{c}\text { Conc. } \\
\text { Posición 1 (\%) }\end{array}$ & $\begin{array}{c}\text { Conc. } \\
\text { Posición 2 (\%) }\end{array}$ & $\begin{array}{c}\text { Conc. } \\
\text { Posición 3 (\%) }\end{array}$ \\
\hline H2 & 14,49 & 7,53 & 8,455 & 9,821 \\
\hline O2 & 2,45 & 0,485 & 0,28 & 0,46 \\
\hline CO & 21,00 & 7,640 & 3,73 & 0,53 \\
\hline N2 & 54,56 & 66,59 & 65,75 & 68,00 \\
\hline CH4 & 0,50 & 0,715 & 0,803 & 0,789 \\
\hline CO2 & 7,00 & 17,04 & 20,99 & 20,40 \\
\hline
\end{tabular}

Fuente: Elaboración Propia.

que al usar aire como agente oxidante, se produce un gas con altas concentraciones de $\mathrm{N}_{2}$ y con bajo valor energético, esto se debe a que uno de los factores que aumenta las concentraciones de $\mathrm{H}_{2}$ y $\mathrm{CO}$ son los agentes oxidantes como el vapor y el $\mathrm{O}_{2}$, lo que mejora el poder calorífico. Para el caso del $\mathrm{O}_{2}$ hace referencia a que este no tiene $\mathrm{N}_{2}$ diluido, hay menor cantidad de impurezas y humedad.

En el proceso de gasificación se tuvo en cuenta tres posiciones de válvula, cuando está totalmente abierta la temperatura es mayor, el contenido de $\mathrm{H}_{2}$ aumenta, simultáneamente la concentración de $\mathrm{CH}_{4}$ disminuye.

\section{Poder calorífico del gas de síntesis}

Teniendo en cuenta la Tabla 10, se puede notar que el mayor poder calorífico del gas producto de la gasificación de las briquetas se dio en la posición 1, válvula semicerrada, esto debido a que, a menor cantidad de aire, la temperatura disminuye, por tanto, hay menos presencia de $\mathrm{H}_{2}$, y aumentan las 
Tabla 10. Poder calorífico del syngas en diferentes posiciones de válvula.

\begin{tabular}{|c|c|c|c|}
\hline \multicolumn{4}{|c|}{ Poder calorífico del gas de las briquetas } \\
\hline $\begin{array}{c}\text { Poder } \\
\text { calorífico }\end{array}$ & $\begin{array}{c}\text { Posición } \\
\mathbf{1}\end{array}$ & $\begin{array}{c}\text { Posición } \\
\mathbf{2}\end{array}$ & $\begin{array}{c}\text { Posición } \\
\mathbf{3}\end{array}$ \\
\hline $\mathrm{MJ} / \mathrm{kmol}$ & 44,67 & 36,8 & 27,56 \\
\hline $\mathrm{kJ} / \mathrm{kg}$ & 1556,23 & 1265,75 & 934,88 \\
\hline $\mathrm{kJ} / \mathrm{Nm}^{3}$ & 2155,44 & 1753,11 & 1294,8 \\
\hline
\end{tabular}

Fuente: Elaboración Propia.

concentraciones de $\mathrm{CO}_{2}$ y $\mathrm{CH}_{4}$. Se puede apreciar que el PCI es de $1556,228 \mathrm{~kJ} / \mathrm{kg}$, y se encuentra en los gases de bajo poder energético [13].

\section{Potencia de generación del gas de síntesis}

En la Tabla 11 se puede observar que el proceso de gasificación y los cálculos correspondientes permitieron obtener como resultado una potencia de $11,8 \mathrm{~kW}$ para el syngas, con una eficiencia en el proceso del $76 \%$, lo cual era esperado debido a que la planta gasificadora usada maneja eficiencias por encima del $75 \%$. Haciendo una relación con la gasificación del trabajo realizado por [14] donde gasifican carbón vegetal (algarrobo), el cual arroja una potencia de 17,37 kW notamos que es mayor, debido a su PCI, es tres veces mayor al obtenido en este trabajo, la potencia de generación no es considerablemente distante teniendo en cuenta la eficiencia del proceso, el tipo de gasificador usado ANKU WBG 10, lo que muestra una menor capacidad del carbón vegetal para la gasificación. Los resultados de la gasificación mostraron que la potencia de generación del gas, supliría el consumo aproximado de dos días de una casa promedio en Colombia, el cual es de $5,1 \mathrm{kWh}$ por día [15].

Tabla 11. Características obtenidas del Syngas.

\begin{tabular}{|c|c|c|c|c|}
\hline \multicolumn{5}{|c|}{ Características de syngas } \\
\hline & $\begin{array}{c}\text { Flujo } \\
\text { másico } \\
(\mathbf{k g} / \mathbf{h})\end{array}$ & $\begin{array}{c}\text { Eficiencia } \\
(\%)\end{array}$ & $\begin{array}{c}\text { Poder } \\
\text { calorícifo } \\
(\mathbf{k j} / \mathbf{k g})\end{array}$ & $\begin{array}{c}\text { Potencia } \\
(\mathbf{k w})\end{array}$ \\
\hline SYNGAS & 35,8 & 76 & 1556 & 11,8 \\
\hline
\end{tabular}

Fuente: Elaboración Propia.

\section{CONCLUSIONES}

De acuerdo, al análisis próximo y elemental se concluye que la elaboración de briquetas con $100 \%$ de estiércol de ganado bovino, no es viable debido al alto contenido de cenizas que este presenta.

En la maquina briquetadora usada, se observa que no es factible elaborar briquetas con contenido de serrín de acacia superiores al 50\% bajo las características de la biomasa y las condiciones de operación establecidas.

Se confirma que la WBT no es una prueba para caracterizar, sino para comparar, puesto que los factores externos influyen en los resultados.

Todas las briquetas elaboradas cumplen con los parámetros de manejabilidad y transporte establecidos por la norma NTC 2060.

La mezcla más favorable para la elaboración de briquetas de serrín de acacia - estiércol de ganado bovino corresponde a $60 \%-40 \%$ respectivamente.

El proceso de gasificación de briquetas con la mezcla más favorable de serrín de acacia - estiércol de ganado bovino, permitió hallar las concentraciones de $\mathrm{H}_{2}, \mathrm{~N}_{2}, \mathrm{CH}_{4}, \mathrm{CO}, \mathrm{CO}_{2 y} \mathrm{O}_{2}$ y posteriormente su PCI, el cual según [13] es un gas de baja calidad.

Los resultados de la gasificación también permitieron concluir que la potencia de generación del gas, supliría el consumo aproximado de dos días de una casa promedio en Colombia, el cual es de 5,1 kWh por día [15].

\section{REFERENCIAS}

[1] "Forestal situation in latinamerica and the caribbean". FAO. 1999.

[2] G. Bavera y C. Pelafort. "Lectura de la bosta del bovino y su relación con la alimentación". Cursos de Producción Bovina de Carne, FAV UNRC. 2006.

[3] Y. Xin and D. Wang. "Influence of moisture content on cattle manure char properties and its Influence of moisture content on cattle manure char properties and its". Journal of Analytical and Applied Pyrolysis. 2018. 
[4] Estadísticas de ganado en Colombia. ICA. 2016.

[5] R. Bailish and D. Oggle. "The Water Boiling Test (WBT)". 2007.

[6] O. Kuti. "Erformance of Composite Sawdust Briquette Fuel in a Biomass Stove under Simulated Condition". Hiroshima: AU J.T. Vol. 12, pp. 284-288. 2009.

[7] K. Nadadurai and V. Morey. "Natural binders and solid bridge type binding mechanisms in briquettes and pellets made from corn stover and switchgrass. bioresource technology". R. 3, s.l.: bioresource technology. Vol. 101, pp. 1082-1090. 2010.

[8] T.U. Onuegbu, U. Ekpunobi, M.O. Ekeoma and I. Ogbu. "Comparative studies of ignition time and water boiling test of coal and biomass briquettes blend". IJRAS. Vol. 7, pp. 153-159.

[9] E.A. Emerhi. "Physical and combustion properties of briquettes produced from sawdust of three hardwood species and different organic binders". Asaba-Nigeria: s.n. Advances in applied science research. Vol. 2, pp. 236-246. 2011.
[10] Y. Xin and D. Wang. "Its, Influence of moisture content on cattle manure char properties and its Influence of moisture content on cattle manure char properties and. s.l.". Journal of analitycal and applied science. 2018.

[11] Ch. Zhanbin. "Norma Temperature Briquetting Technology for Biomass with Original Moisture Content International Conference on Bioenergy Utilization and Evironmental Protection". Dalian-China: LAMNET WORKSHOP. 2003.

[12] E.G. Fonseca Cuenca y L.F. Tierra Tingo. "Desarrollo de un proceso tecnológico para la obtención de briquetas de aserrín de madera y cascarilla de arroz, y pruebas de producción de gas pobre". Escuela Superior Politécnica de Chimborazo. Riobamba, Ecuador. 2011.

[13] A. Valderrama. "Briquetas de residuos solidos organicos como fuente de energía calorifica en cocinas no convencionales". Lima-Peru: guzlop. 2011.

[14] H. Martins. "Uso de Madeira para fins energético". Belo Horizonte: s.n. 1980.

[15] T. Milne. "Biomass gasifier Tars: Their nature, formation, and conversion". National renewable energy laboratory. 1998. 